\title{
Highly efficient method for Synthesis of benzoquinones using Hypervalent iodine (III) reagent and sodium bisulfate
}

\author{
Kulbhushan A. Sasane, Vikas N. Telvekar* \\ Department of Pharmaceutical Sciences and Technology, \\ Institute of Chemical Technology, Matunga, Mumbai, India \\ * Correspondence: E-mail: vikastelvekar@rediffmail.com
}

\section{Supporting Information:}

\section{GENERAL PROCEDURE}

General procedure for synthesis of benzoquinone derivatives (Table 3, entry 1): (Diacetoxyiodo) benzene $(5 \mathrm{mmol}, 1.61 \mathrm{~g})$ and $\mathrm{NaHSO}_{4} \cdot \mathrm{H}_{2} \mathrm{O}(1 \mathrm{mmol}, 0.138 \mathrm{~g})$ were stirred for 10-15 min, at room temperature in aqueous solution of acetonitrile $(5 \mathrm{ml}$ water and $5 \mathrm{ml}$ acetonitrile). In this reaction mixture benzamide (1mmol) was added and stirring was continuing till reaction goes to completion (TLC). After completion of reaction, the reaction mixture was quenched with water and further extracted with chloroform $(3 \times 10 \mathrm{ml})$. The combined chloroform layers were washed with water $(3 \times 20 \mathrm{ml})$ dried over $\mathrm{Na}_{2} \mathrm{SO}_{4}$, and concentrated on rota-evaporator to get the crude residue. The residue was further purified by column chromatography on silica gel using ethyl acetate: hexane (1:9) as an eluent to afford pure benzoquinone.

\section{Characterization Data of benzoquinone derivatives:}

\section{1, 4-Benzoquinone (Table 2, Entry 1) ${ }^{1}$ :}

$\operatorname{Mp} 115{ }^{\circ} \mathrm{C}\left(\mathrm{Lit}^{[1]} \mathrm{Mp} 112{ }^{\circ} \mathrm{C}\right) ;{ }^{1} \mathrm{H}$ NMR (60 MHz, $\left.\mathrm{CDCl}_{3}\right)$ : 7.59-7.68 (s, 4H); IR (KBr): $3165,3070,1665,1589,1312,1264,897$ 
2-Methyl-1, 4-benzoquinone (Table 2, Entry 2)²:

Mp 68-69 ${ }^{\circ} \mathrm{C}\left(\operatorname{Lit}^{[2]} \mathrm{Mp} 69^{\circ} \mathrm{C}\right)$; IR (KBr): 3728, 3021, 2317, 1656, 1604, 1452, 1218, 904, 757. ${ }^{1} \mathrm{H}$ NMR $60 \mathrm{MHz}\left(\mathrm{CDCl}_{3}\right): \delta 2.04(\mathrm{~s}, 3 \mathrm{H}), 6.56(\mathrm{~s}, 1 \mathrm{H}), 6.65-6.70(\mathrm{~m}, 2 \mathrm{H})$.

2-Chloro-1, 4-benzoquinone (Table 2, Entry 3) ${ }^{3}$ :

Mp 54-56 ${ }^{\circ} \mathrm{C}\left(\mathrm{Lit}^{[3]} \mathrm{Mp} \mathrm{55-56}{ }^{\circ} \mathrm{C}\right) ;{ }^{1} \mathrm{H}$ NMR $60 \mathrm{MHz}\left(\mathrm{CDCl}_{3}\right): \delta 6.80(\mathrm{~d}, 1 \mathrm{H}), 7.3(\mathrm{~d}, 1 \mathrm{H})$, $6.73(\mathrm{~s}, 1 \mathrm{H})$.

1, 4-benzoquinone (Table 2, Entry 4) ${ }^{4}$ :

Mp 114-116 ${ }^{\circ} \mathrm{C}\left(\operatorname{Lit}^{[4]} \mathrm{Mp} 115^{\circ} \mathrm{C}\right) ;{ }^{1} \mathrm{H}$ NMR $300 \mathrm{MHz}\left(\mathrm{CDCl}_{3}\right): \delta 3.81$ (s, 3H), 5.93 (br s, 1H), 6.71 (br s, 2H).

1, 4 benzoquinone (Table 2, Entry 5) ${ }^{5}$ :

$\operatorname{Mp} 115{ }^{\circ} \mathrm{C}\left(\right.$ Lit $\left.{ }^{[5]} \mathrm{Mp} 115{ }^{\circ} \mathrm{C}\right) ;{ }^{1} \mathrm{H}$ NMR (60 MHz, $\left.\mathrm{CDCl}_{3}\right)$ : 7.59-7.68 (s, 4H); IR (KBr): $3165,3070,1665,1589,1312,1264,897$

1, 4 benzoquinone (Table 2, Entry 7) ${ }^{6}$ :

$\operatorname{Mp} 115^{\circ} \mathrm{C}\left(\right.$ Lit $\left.^{[6]} \mathrm{Mp} 111{ }^{\circ} \mathrm{C}\right) ;{ }^{1} \mathrm{H}$ NMR (60 MHz, $\left.\mathrm{CDCl}_{3}\right)$ : 7.58-7.68 (s, 4H); IR (KBr): $3165,3070,1665,1589,1312,1263,897$

4-Hydroxy-2, 4, 6-trimethylcyclohexa-2, 5-dienone (Table 2, Entry 8) ${ }^{7}$ :

Mp 45-46 ${ }^{\circ} \mathrm{C}\left(\mathrm{Lit}^{[7]} \mathrm{Mp} 45-46{ }^{\circ} \mathrm{C}\right) ;{ }^{1} \mathrm{H}$ NMR $300 \mathrm{MHz}\left(\mathrm{CDCl}_{3}\right): \delta 1.41$ (s, 3H), $1.84(\mathrm{~s}, 6 \mathrm{H})$, 6.62 (br s, $2 \mathrm{H}) ;{ }^{13} \mathrm{C}$ NMR $\delta 187.0,147.9,132.9,66.8,26.9,15.7$ 


\section{2-Methoxy-1, 4-benzoquinone (Table 2, Entry 9) ${ }^{8}$ :}

Mp 142-144 ${ }^{\circ} \mathrm{C}\left(\operatorname{Lit}^{[8]} \mathrm{Mp} 142{ }^{\circ} \mathrm{C}\right) ;{ }^{1} \mathrm{H}$ NMR $300 \mathrm{MHz}\left(\mathrm{CDCl}_{3}\right): \delta 3.81$ (s, 3H), 5.93 (br s, $1 \mathrm{H}), 6.71(\mathrm{br} \mathrm{s}, 2 \mathrm{H})$

4-Hydroxy-4-methylcyclohexa-2, 5-dienone (Table 2, Entry 10) ${ }^{9}$ :

Mp 77-78 ${ }^{\circ} \mathrm{C}\left(\right.$ Lit $\left.{ }^{[9]} \mathrm{Mp} 74{ }^{\circ} \mathrm{C}\right)$; IR (KBr) 3411, 2974, 2921, 1674, 1644, $1372 \mathrm{~cm}-1 ;{ }^{1} \mathrm{H}$ $\operatorname{NMR}\left(\mathrm{CDCl}_{3}, 300 \mathrm{MHz}\right) \delta 1.46(\mathrm{~s}, 3 \mathrm{H}), 2.02(\mathrm{~s}, 1 \mathrm{H}), 6.12(\mathrm{~d}, 2 \mathrm{H}), 6.88(\mathrm{~d}, 2 \mathrm{H}) ;{ }^{13} \mathrm{C} \mathrm{NMR}$ $\left(\mathrm{CDCl}_{3}, 100 \mathrm{MHz}\right) \delta 14.1(\mathrm{q}), 17.0(\mathrm{t}), 42.0(\mathrm{t}), 70(\mathrm{~s}), 128.2(\mathrm{~d}), 151.4(\mathrm{~d}), 185.7(\mathrm{~s})$

\section{References:}

1. Derikvand, F.; Bigi, F.; Maggi, R.; Piscopo, C. G.; Sartori, G. Oxidation of hydroquinones to benzoquinones with hydrogen peroxide using catalytic amount of silver oxide under batch and continuous-flow conditions. Journal of Catalysis 2010, 271, 99-103.

2. Zagulyaeva, A. A.; Banek, C. T.; Yusubov, M. S.; Zhdankin, V. V. Hofmann rearrangement of Carboxamides mediated by hypervalent iodine species generated in situ from Iodobenzene and oxone: reaction scope and limitations. Org. Lett. 2010, 12, $4644-4647$.

3. Zagulyaeva, A. A.; Banek, C. T.; Yusubov, M. S.; Zhdankin, V. V. Hofmann Rearrangement of Carboxamides Mediated by Hypervalent Iodine Species Generated in Situ from Iodobenzene and Oxone: Reaction Scope and Limitations. Org. Lett. 2010, 12, 4644-4647.

4. Derikvand, F.; Bigi, F.; Maggi, R.; Piscopo, C. G.; Sartori, G. Oxidation of hydroquinones to benzoquinones with hydrogen peroxide using catalytic amount of 
silver oxide under batch and continuous-flow conditions. Journal of Catalysis 2010, 271, 99-103.

5. Telvekar, V. N.; Takale, B. S. Carbon-carbon cleavage of aryl diamines and quinone formation using sodium periodate: a novel application. Tetrahedron.Lett.2010, 51, 3940-3943.

6. Villemin, D.; Hammadi, M.; Hachemi, M. Supported metalated phthalocyanine as catalyst for oxidation by moleculer oxygen. Sythesis of quinones and carbonyl compounds. Synth. Comm. 2002, 32, 1501-15115

7. Zagulyaeva, A. A.; Banek, C. T.; Yusubov, M. S.; Zhdankin, V. V. Hofmann Rearrangement of carboxamides mediated by hypervalent iodine species generated in situ fromilodobenzene and oxone: reaction scope and limitations. Org. Lett. 2010, 12, $4644-4647$.

8. Parker, K. A. Spero, D. M.; Koziski, K. A. Evaluation of some preparations of trialkoxyphthalic acid derivatives. J. Org. Chem. 1987, 52, 183-188.

9. Wartchow, R.; Winterfeldt, E.; Wong, Y. S. New cyclohexadienone derivatives: preparation and chiral discrimination in high-pressure diels alder cycloadditions. Chem. Eur. J. 2001, 7, 2349 - 2369. 